\title{
Annealing behavior of quench-condensed hydrogen and deuterium films
}

\author{
Uwe Albrecht, Peter Evers and Paul Leiderer \\ Fakultät für Physik, Unizersität Konstanz, D-7750 Konstanz, Ciermany
}

Received 20 April 1992; accepted for pubication 29 June 1992

We have studied the annealing behavior of thin molecular hydrogen and deuterium films (typicat thickness $10 \mathrm{~nm}$ ) prepared by quench-condensation at $1.5 \mathrm{~K}$ on a silver substrate. Changes in the optical thickness and the heterogeneity of the films were monitured using optically excited surface plasmons. Upon annealing the films exhibit an irreversible change from a homogeneous to a strongly heterogeneous structure on a length scale of some bundred $\mathrm{nm}$, accompanied by an apparent loss in optical thickness. This behavior is interpreted as being due to the formation of large bulk crystallites on the surface, resulting from the incomplete wetting of solid hydrogen on silver.

\section{Introduction}

The properties of quench-condensed films have been a topic attracting increasing attention over the past years. Their structure and excitations may differ considerably from films in thermal equilibrium. Atoms and molecules exhibiting simple van der Waals-type intermolecular interaction, like rare gases and the hydrogen isotopes, have been used as models to study the properties of these systems, using phonon scattering [1], $\mathrm{X}$-ray diffraction [2], surface state electrons [3] and heat capacity measurements $[2,4]$. Generally the films are found to be strongly disordered.

We have employed a new technique using optically excited surface plasmons to study how the relaxation towards the thermodynamic equilibrium in these films proceeds upon thermal annealing.

\section{Experimental}

Surface plasmons (SP) are optically excited on a thin silver film using the Kretschmann configuration [5]. The silver, serving also as substrate for the quench-condensation, is thermally evaporated onto the base of a glass prism. We illuminate the silver film from the glass side with a HeNe-laser incident at a particular angle $\Theta_{R}$, where the surface plasmons are resonantly excited. This leads to a sharp drop in the totally reflected light intensity at this resonance angle. Depositing a thin dielectric layer on the silver surface changes the SP resonance condition. The resulting shift in $\Theta_{R}$ is a measure for the optical thickness of the dielectric material.

Fig. 1 shows a calculation of $\Theta_{R}$ versus the optical thickness $d$ of a $\mathrm{H}_{2}$-layer deposited on silver (using a refractive index of $\varepsilon_{\mathrm{H}}=1.295$, i.e. assuming bulk density). Up to about $d=1000 \AA$ the shift in $\Theta_{R}$ is in good approximation proportional to the layer thickness. Towards larger values of $d$ it gets less sensitive due to the exponential decay of the SP field into the adjacent halfspace. By accurately monitoring the shift in resonance angle we can thus directly measure the optical thickness of thin films deposited on the silver surface carrying the SP wave. In addition, we simultaneously determine the halfwidth in angle $\Delta \theta$ of the SP resonance, which increases when the SP wave scatters from inhomogeneities 


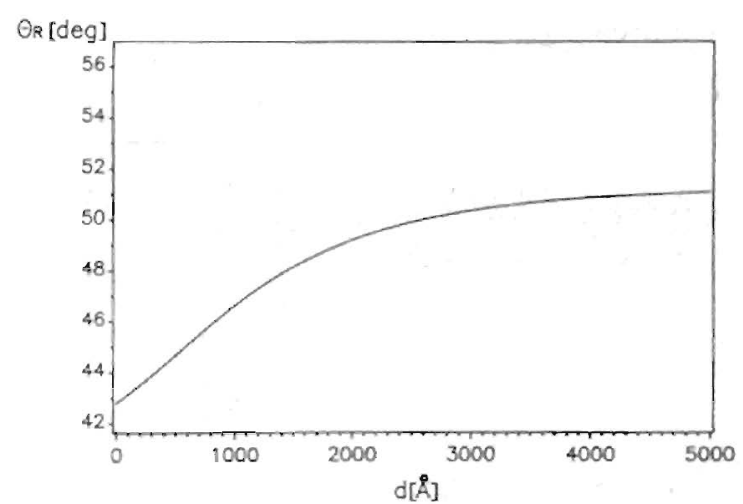

Fi.. 1. Calculated surface plasmon resonance angle $\Theta_{\mathrm{R}}$ on silver versus thickness of an additionally deposited $\mathrm{H}_{2}$-film (bulk density is assumed).

in the film. Hence this provides a measure for structural changes within the dielectric film. Details of the measuring technique are described elsewhere [6].

The hydrogen films were quench-condensed onto the surface at $\sim 1.5 \mathrm{~K}$; the temperature of the gas entering the experimental cell was below $25 \mathrm{~K}$. A low temperature of the incoming gas is crucial for the formation of disordered quenchcondensed films [7]. We annealed the films at a rate of $0.5 \mathrm{~K} / \mathrm{h}$ for the experiments shown below. Measurements at slower rates proved the results to be qualitatively independent of the annealing rate. The films were not in equilibrium with the vapor, since cold surfaces close to the prism in the experimental cell acted as cryopumps for desorbing gas molecules.

\section{Results}

Fig. 2 shows the results of an annealing experiment on a quench-condensed $\mathrm{H}_{2}$-film of $\sim 100 \AA$ thickness. The shift in $\Theta_{\mathrm{R}}$ (plotted as optical thickness $d$, assuming bulk density) and the resonance width $\Delta \Theta$ (normalized to the value on bare silver) are plotted versus the annealing temperature. The film is initially homogeneous on a length scale of the order of the light wavelength, since the plasmon resonance width is not significantly

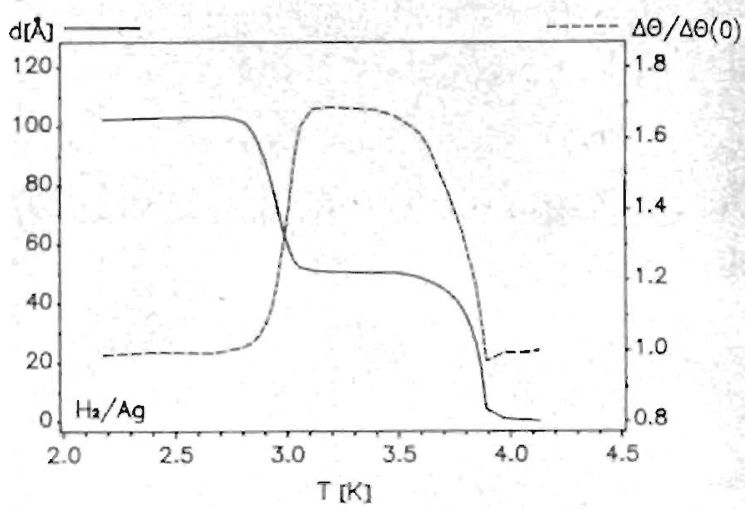

Fig. 2. Annealing behavior of a quench-condensed $\mathrm{H}_{2}$-film. The thickness $d$ (solid line) is calculated using the relation in fig. 1. The dashed curve shows the simultaneously measured width of the surface plasmon resonance $\Delta \theta$, normalized to the value on bare silver $\Delta \Theta(0), T$ is the annealing temperature.

different from the value of the bare silver substrate. Between 2.5 and $3 \mathrm{~K}$, however, there is a strong increase in SP scattering, accompanied by a drastic shift of $d$ to about half its initial value. This state then prevails until the desorption of the hydrogen molecules sets in (slightly below 4 $\mathrm{K})$. The annealing effect is a function of film thickness, being less pronounced for thin films than for thick films (the relative decrease in $d$ for a film of $35 \AA$ amounts to only $\sim 20 \%$ ). All changes in $d$ and $\Delta \Theta$ induced by the annealing are irreversible. Interrupting the annealing process at any point and subsequently lowering the

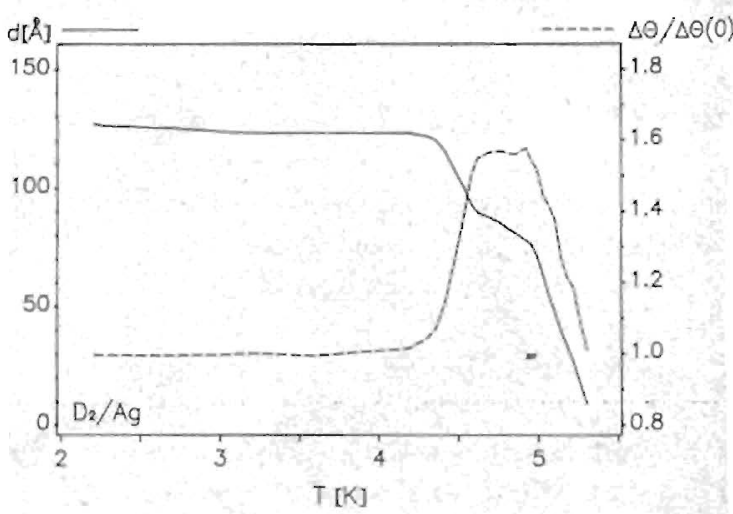

Fig. 3. Annealing behavior of a quench-condensed $D_{2}$-film. 
It remains unclear, why we do not see annealing effects in the quench-condensed neon films, which also exhibit triple point wetting on silver [12]. Possibly the surface diffusion on neon is not fast enough at temperatures below the desorption threshold.

\section{Conclusion}

We have investigated the annealing behavior of thin quench-condensed films of the hydrogens by monitoring the resonance angle of optically excited surface plasmons and their resonance width as measures for the optical film thickness and heterogeneity. Upon annealing the initially homogeneous films exhibit a transition to a state which is strongly heterogencous on the scale of the light wavelength, accompanied by a pronounced decrease in resonance angle. The observations can be consistently explained by a dewetting process involving the formation of large bulk crystallites on a thin wetting film of monolayer thickness. The rearrangement of the molecules involved can only occur via surfacc diffusion. Recent measurements [11] indicate that surface melting might be the mechanism leading to the high mobilities necessary.

\section{Acknowledgements}

This work has been supported by the SFB306 of the Deutsche Forschungsgemeinschaft. We appreciate the help of $\mathrm{R}$. Conradt with the experiments.

\section{References}

[1] T. Klitsner and R.O. Pohl, Phys. Rev B 34 (1986) 6045

[2] N. Sieinmetz, H. Menges, J. Dutzi, H. v. Löhneysen and W. Goldacker. Phys. Rev. B 39 (1989) 2838.

[3] K. Kono, U. Albrecht and P. Leiderer, J. Low Temp. Phys. 82 (1991) 279.

[4] R.B. Phelps. J.T. Birmingham and P.L Richards. 10 be published.

[5] E. Kretschmann and H. Raether, Z. Naturforsch. 23a (1968) 2135.

16) U. Albrecht, H. Dilger, P. Evers and P. Leiderer, in: Process Module Metrology, Control and Clustering, Eds. C.J. Davis, I.P. Herman and T.R. Turner, Proc. SPIE 1594 (1992) 344.

[7] K.H. Müller, Surf. Sci 184 (1987) L375.

[8] A.D. Migone, A. Hoffmann, J.G. Dash and O.E. Vilches. Phys.Rev. B 37 (1.988) 5440.

[9] G. Reiter, Phys. Rev. Lett. 68 (1992) 75.

[10] I.F. Silvera, Rev. Mod. Phys. 52 (1980) 393.

[11] M. Maruyama, M. Bienfait, I'.C. Liu, Y.M. Liu, O.E. Vilches and R. Rieutord. Phys. Rev. Letl, to be pub. lished.

[12] A.D. Migone, J.G. Dash, M. Schick and O.E. Vilches. Phys. Rev, B 34 (1986) 6322. 